\title{
Nuss bar procedure: past, present and future
}

\author{
Donald Nuss, Robert J. Obermeyer, Robert E. Kelly \\ Department of Surgery, Eastern Virginia Medical School, 601 Children'S Lane, Norfolk, Virginia 23507, USA \\ Correspondence to: Donald Nuss, M.B., CH.B. Children'S Hospital of The King'S Daughters, Pediatric Surgery, 601 Children'S Lane, Norfolk, \\ Virginia 23507, USA. Email: nussdonald@gmail.com.
}

\begin{abstract}
Repair of pectus excavatum began at the beginning of the $20^{\text {th }}$ century before endotracheal intubation was standard practice. Surgeons therefore developed techniques that corrected the deformity using an open procedure via the anterior chest wall. Initial techniques were unsatisfactory, but by the 1930 s the partial rib resection and sternal osteotomy technique had been developed and was used in combination with external traction post-operatively to prevent the sternum from sinking back into the chest. In 1949, Ravitch recommended complete resection of the costal cartilages and complete mobilization of the sternum without external traction, and in 1961 Adkins and Blades introduced the concept of a substernal strut for sternal support. The wide resection resulted in a very rigid anterior chest wall, and in some instances, the development of asphyxiating chondrodystrophy. The primary care physicians therefore became reluctant to refer the patients for repair. In 1987, Nuss developed a minimally invasive technique that required no cartilage or sternal resection and relied only on internal bracing by means of a sub-sternal bar, which is inserted into the chest through two lateral thoracic incisions and guided across the mediastinum with the help of thoracoscopy. After publication of the procedure in 1998, it became widely accepted and a flood of new patients suddenly started to appear, which allowed for rapid improvements and modifications of the technique. New instruments were developed specifically for the procedure, complications were recognized, and the steps taken to prevent them included the development of a stabilizer and the use of pericostal sutures to prevent bar displacement. Various options were developed for sternal elevation prior to mediastinal dissection to prevent injury to the mediastinal structures, allergy testing was implemented, and pain management improved. The increased number of patients coming for repair permitted studies of cardiopulmonary function, which showed that patients with a severe degree of pectus excavatum have right- sided cardiac compression, decreased filling, and decreased stroke volume. The degree of pulmonary restriction and obstruction is related to the degree of deformity and degree of cardiac displacement into the left chest. The indications for surgical repair have been clearly outlined, the procedure has been standardized, and post-operative management protocols are now available. A review of our prospective database showed that $98 \%$ of patients have a good to excellent outcome. This review of the "Past" outlines the progression of the surgical techniques during the $20^{\text {th }}$ century, the review of the "Present" outlines the important modifications and results of the closed technique, and the review of the "Future" outlines the various new options that are becoming available for the treatment of pectus excavatum.
\end{abstract}

Keywords: Pectus excavatum; minimally invasive repair; history; technique; post-operative management

Submitted Feb 09, 2016. Accepted for publication Mar 16, 2016.

doi: 10.21037/acs.2016.08.05

View this article at: http://dx.doi.org/10.21037/acs.2016.08.05

\section{Past}

Anesthesia using an ether mask was a landmark development in 1846; however, it did not provide a safe airway for thoracic surgery. For this reason, thoracic surgery remained in its infancy until endotracheal anesthesia was introduced in the 1920's $(1,2)$. It is therefore not surprising that initial attempts to correct pectus excavatum were designed to avoid opening the chest and to correct the deformity via an 
incision on the anterior chest wall. In 1911, Meyer removed two rib cartilages on the right side but concluded that the result was unsatisfactory (3). In 1913, Sauerbruch resected the depressed section of the anterior chest wall which relieved the patient's cardiac compression, palpitations, and blackouts, allowing him to return to work, but left him with acquired Poland's syndrome. Sauerbruch similarly considered this result to be unsatisfactory (4). Therefore, in the 1920's, when confronted by a young girl with a severe pectus excavatum he developed the technique of only partial cartilage resection and sternal osteotomy which is now frequently referred to as a "modified Ravitch technique" (5). In order to prevent the sternum from sinking back into the chest, Sauerbruch used external traction by passing a steel wire through the sternum and attaching it to an orthopedic traction device for several weeks.

In 1949, Ravitch popularized an approach which avoided the need for external traction and that included radical resection of all the costal cartilages, complete disconnection of the sternum from the chest wall and a wedge osteotomy of the sternum (6). He advocated doing the procedure in infants and pre-school age children, which resulted in a very rigid and corrugated chest wall, and sometimes, damage to the growth centers, causing the development of acquired asphyxiating chondrodystrophy in later years. As pediatricians provided most of the longitudinal care of these patients, they were the first to recognize this problem and referrals for pectus excavatum repair significantly decreased during this period (7).

In 1956, Wallgren and Sulamaa developed a novel strategy of internal support to prevent sternal regression by placing a subcutaneous bar across the chest that went through the body of the sternum (8). In 1961, Adkins and Blades went one step further and placed the bar behind the sternum (9). These surgeons were essentially responsible for the standard rib resection, sternal osteotomy, and substernal support method of pectus excavatum repair during the subsequent 40 years.

Thoracic surgeons and trauma surgeons had noted the flexibility and malleability of the chest wall for a considerable period of time, as noted by Samuel Kelly in 1929: "dislocations of the sternum and of the ribs are very unusual in children, owing to the softness and elasticity of the chest walls" (10). This principle was reasserted by Haller in 1986 when he stated: "the flexible chest of a child makes disruption of the chest wall unlikely" and by Wesson in 1998: "Chest wall injuries in children require substantial impact to a pliable chest wall" $(11,12)$.
By the mid 1980's Nuss had become disillusioned with the results of the wide resection technique, not only in patients seen in his own practice, but also in patients operated on at other centers who came for a "second opinion" seeking help for a poor outcome. He therefore realized that another option needed to be found.

He was well aware of the "soft, elastic, flexible and pliable chest of young children" as well as the admonition in The Textbook of Advanced Pediatric Life Support that "only two fingers should be used for cardiac compression" to avoid crushing the heart (13). He therefore could see no reason for excising these soft and malleable structures. In addition to this knowledge, he knew that there was an avascular sub-sternal plane from the experience gained as a result of performing sub-sternal colon interpositions, median sternotomy, and placement of Adkins struts.

When, in 1987, a 4-year-old male patient presented with a severe pectus excavatum, he discussed with the parents the option of not removing the chest wall structures. They allowed him to proceed with his proposed surgical solution which included placing a bar under the sternum without cartilage resection or sternal osteotomy. The procedure was uneventful and gave an excellent correction.

Several key innovations were developed in the following two decades. The first one was to strengthen the bar as the original one was not strong enough to provide a durable correction. The company that made the original bar agreed to make it stronger, but after two years stated that they could no longer accept the risk involved and stopped making it. Fortunately, another company agreed to partner with Nuss on the project and not only was the bar completely redesigned, but new instruments were designed and developed specifically for the minimally invasive repair of pectus excavatum (MIRPE) $(14,15)$. The incision was moved from the anterior chest wall to bilateral lateral chest wall incisions.

Other important additions included the use of thoracoscopy in 1998, which made the substernal dissection safer and helped optimize bar placement. Bar displacement proved to be a major problem and therefore a stabilizer was developed in 1998 to improve bar fixation (15). However the addition of pericostal sutures in 2002 reduced bar displacement to less than $1 \%$ when combined with the stabilizer (16). Park has also developed several new devices such as a "claw fixator" and "hinge plate" to help prevent bar displacement (17).

Improvements in the substernal dissection have been crucial to prevent cardiac injury during this part of the 


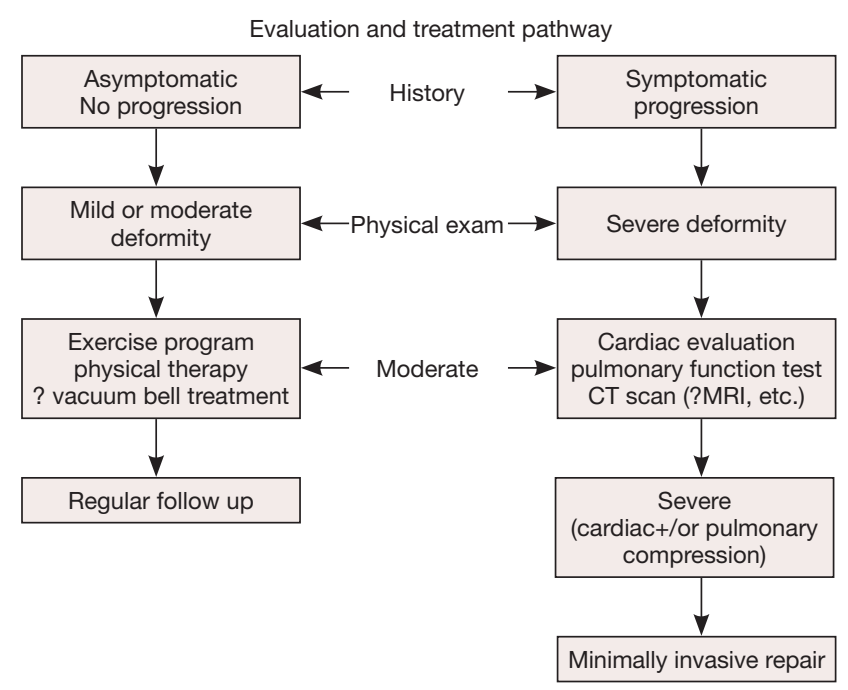

Figure 1 Evaluation and treatment algorithm.

procedure. Initially, a more cephalad tunnel was created superior to the deepest point of the defect with one tunneler, before passing a second tunneler under the deepest point. Later, sternal elevation prior to substernal dissection was developed and found to be facilitated by using a variety of retractors, towel clamps, Klobe's suction cup, or Park's "Crane Technique" (16,17).

\section{Surgical indications}

Initially there were no criteria to determine which patients had a deformity severe enough to warrant surgical correction. A treatment algorithm was developed which took into account the following: symptoms, severity and phenotype of the deformity, deformity progression, objective study results (computed tomography (CT) scan index, electrocardiogram (EKG), echocardiogram), and Pulmonary Function Tests (18) (Figure 1).

This algorithm resulted in $33 \%$ to $50 \%$ of the patients being selected for surgical correction, while the other 50 to $66 \%$ were started on an exercise and posture program and re-evaluated at yearly intervals. More recently these patients have been offered treatment with the Vacuum Bell $(19,20)$

The advent of the MIRPE resulted in a dramatic increase in the number of patients presenting for repair $(15,21)$. This large increase in the numbers of patients undergoing repair has allowed many centers to develop technical refinements, thereby making the procedure even safer and has allowed numerous pre-and post-operative studies to be performed, showing that pectus excavatum causes significant cardiac and pulmonary compression and dysfunction which is markedly improved after repair (22-24).

\section{Present}

\section{Pre-operative evaluation}

Patients are evaluated to see if they are candidates for surgical correction based on the abovementioned criteria (Figure 1).

Patients are deemed suitable candidates for MIRPE if they manifest two or more of the following criteria:

(I) The medical history reveals that the patient is symptomatic. The most common complaints are shortness of breath with exercise, lack of endurance, and chest pain. Frequently there is also a history of progression of the deformity, especially during puberty;

(II) Physical exam shows that there is a moderate to severe pectus excavatum deformity which may be symmetric or asymmetric;

(III) The chest wall imaging [CT or magnetic resonance imaging (MRI)] shows a severe pectus excavatum deformity defined as a Haller CT index higher than 3.2 or correction index greater than $10 \%$ (25), cardiac and/or pulmonary compression or displacement;

(IV) Pulmonary Function Studies demonstrate a restrictive or obstructive pattern;

(V) Cardiology evaluation elucidates cardiac compression or displacement, rhythm disturbance, and/or mitral valve prolapse;

(VI) Poor body image and psycho-social maladjustment.

If the patient has a mild deformity and does not fulfill the abovementioned criteria for surgical correction, then he/she will be started on a deep breathing with breath holding and posture exercise program, and may be offered non-operative treatment with the Vacuum Bell $(19,20)$. These patients are typically re-evaluated every 3 to 6 months to ensure that they are complying with the conservative treatment and to check whether the deformity is improving or progressing.

The patients who fulfill the criteria for MIRPE are also started on the deep breathing and posture exercise program. They are checked for metal allergy and their chest is measured between bilateral mid-axillary lines to determine the length of the bar to be inserted. The bar should be about 1 inch ( 2.5 centimeters) shorter than the measurement from the bilateral mid-axillary lines. 


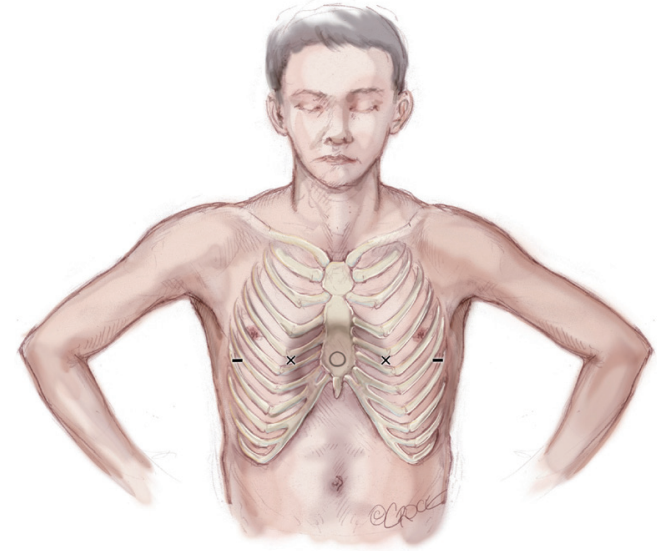

Figure 2 Shows how the chest is marked before starting surgery. The deepest point of the depression is marked with a circle, the bilateral incision sites are marked with straight lines and the thoracic entry and exit sites are marked with an "X", all of which are in the same horizontal plane.

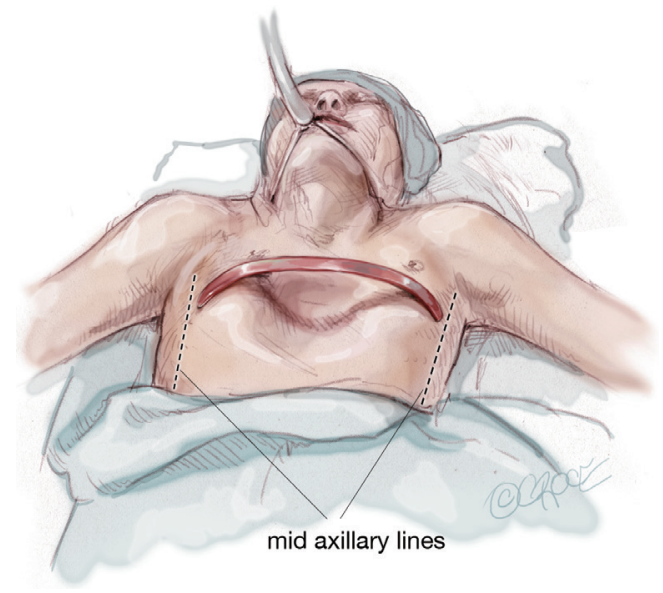

Figure 3 The length of the bar is determined by measuring from right to left mid-axillary line and subtracting $2.5 \mathrm{~cm}$ (1 inch) from that measurement.

\section{Surgical technique}

After induction of general endotracheal anesthesia and insertion of an indwelling bladder catheter, the patient is positioned supine with both arms abducted approximately 70-degrees at the shoulder. The chest is carefully palpated and the deepest point of the depression is marked with a circle using a marking pen. Next the intercostal spaces that are in the same horizontal plane as the deepest point of the excavatum are marked with an " $\mathrm{X}$ " just inside or medial to the top ridge of the depression. That is just medial to where the chest starts to cave in on each side. Finally, the incision sites are marked laterally between the anterior axillary line and the mid axillary line in the same horizontal plane as the deepest point of the depression (Figure 2).

The chest is measured from right mid axillary line to left mid axillary line and a pectus bar is selected based on this measurement, minus $2.5 \mathrm{~cm}$ ( 1 inch). Pilegaard has advocated the use of a shorter and eccentrically placed bar with the stabilizer in a more medial position, but this can only be used in patients who have completed their growth spurt (26). The bar is bent into a smooth convex shape such that it fits comfortably against the skin leaving a $2-4 \mathrm{~cm}$ flat section in the middle to support the sternum (Figure 3).

An intravenous first-generation cephalosporin is given as prophylaxis and maintained for up to 48 hours postoperatively. The chest is prepared and draped in a sterile manner, taking care to prep the skin down to the posterior axillary line.

Thoracoscopy, using $\mathrm{CO}_{2}$ up to a pressure of $5 \mathrm{mmHg}$, is then carried out by inserting a $5 \mathrm{~mm}$ trocar at the midaxillary line, approximately two interspaces below the proposed right lateral skin incision. It is important to direct the trocar in a superior direction to avoid perforating the diaphragm and penetrating the liver. It is inserted at the mid-axillary line level so that one will be able to advance the thoracoscopy across the mediastinum. A 5-mm scope with a flexible tip is preferable, and if that is not available then a 30-degree scope is helpful. While looking through the thoracoscopy, it is important to check whether the internal anatomy corresponds well with the external markings. In addition, the mediastinum should be evaluated to see whether it will be necessary to elevate the sternum prior to starting the trans-mediastinal dissection. In complex cases, bilateral thoracoscopy may be helpful (Figure 4).

Some centers now use sternal elevation in all cases as it makes the mediastinal dissection easier and safer by markedly decreasing the risk of pericardial or cardiac perforation. There are several options available for sternal elevation before starting the mediastinal dissection. One option is to create an additional tunnel higher up where the depression is not so deep, and leaving that introducer in place to keep the sternum elevated while dissecting under the deepest point of the depression. A second option, in younger patients with a flexible chest, is to elevate the sternum by using the Vacuum Bell. A third option is to elevate the sternum by using a retractor inserted either through an additional sub-xiphoid incision or laterally" $(27,28)$. A fourth option is to elevate the sternum by using 


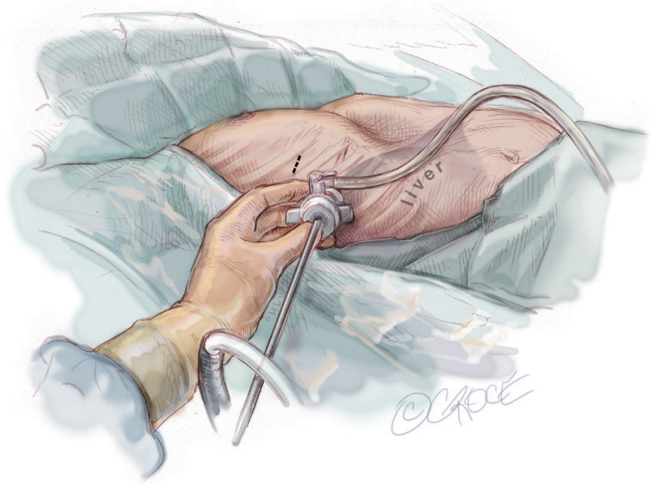

Figure 4 The thoracoscope is inserted in the right mid-axillary line approximately 2 intercostal spaces inferior to the incision site and directed in a superior direction to avoid injuring the diaphragm.

\section{Park's Crane Technique (17,29) (Figure 5).}

Bilateral skin incisions are made at the previously marked sites between the anterior and mid axillary lines. The incisions are carried down to the rib cage and the subcutaneous tunnels are created following the rib cage up to the previously marked " $\mathrm{X}$ " just medial to the top of the ridge. If the planned subcutaneous tunnel is superior to the origin of the pectoralis muscles, then the tunnel should proceed under this muscle.

A retractor is inserted into the tunnel and the introducer is slowly advanced up the tunnel with the tip facing posteriorly. At the previously marked "X", the introducer is gently pushed through the intercostal muscles under direct vision via the thoracoscopy. The introducer is
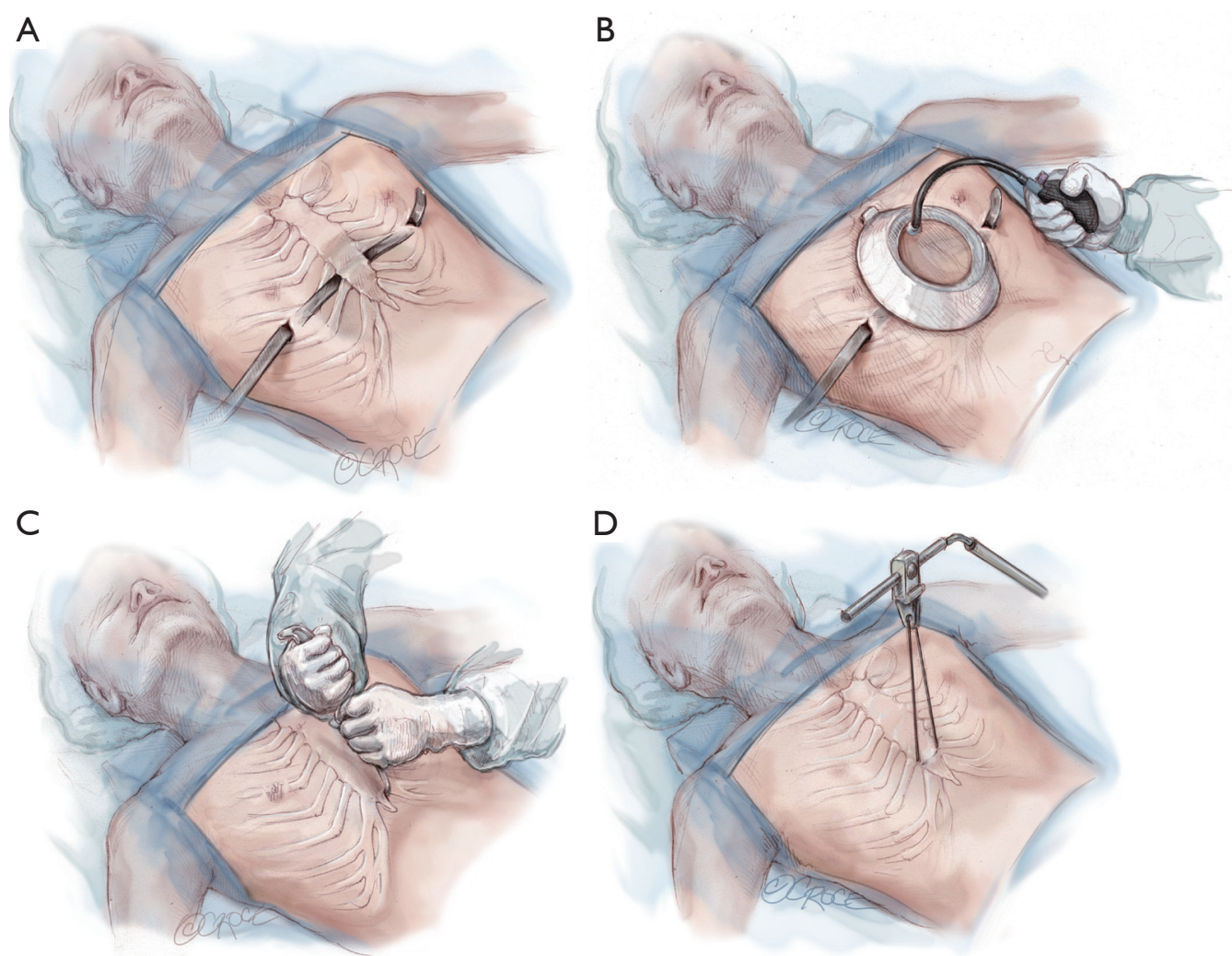

Figure 5 (A) Shows the sub-sternal, trans-thoracic introducer in position. However, in order to minimize the risk of cardiac and pulmonary laceration during dissection, sternal elevation should be accomplished prior to creating the substernal tunnel by means of one of several external elevation techniques; (B) Shows sternal elevation using the vacuum bell to facilitate the mediastinal dissection in young patients with a malleable chest wall; (C) Shows sternal elevation with a hook inserted through a small sub-xiphoid incision; (D) Shows sternal elevation using the Crane technique where a wire is passed through the anterior table of the sternum and attached to a Thompson or Rultract retractor to elevate the sternum. 


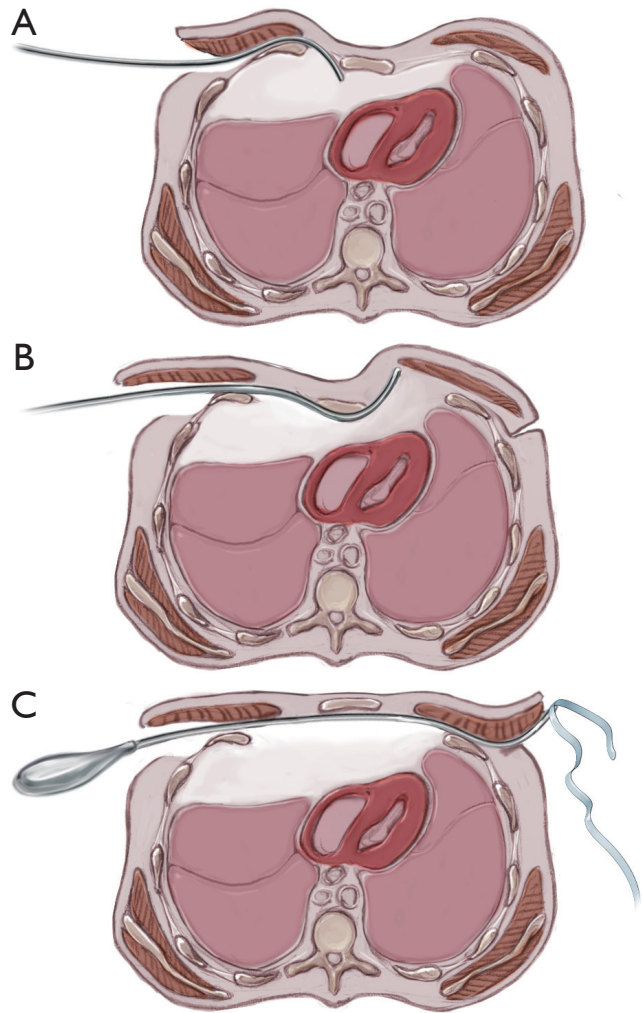

Figure 6 (A) Shows insertion of the introducer through the subcutaneous tunnel, under the pectoralis major muscle before entering the thoracic cavity at the previously marked "X"; (B) Shows the introducer passing immediately under the sternum with the tip always in view and using a "pawing" motion for dissection; (C) Shows the introducer having emerged from the thorax through the left intercostal spaced marked with an " $\mathrm{X}$ " and having passed under the left pectoralis muscle and out through the left subcutaneous tunnel.

turned over so that the tip faces anteriorly and very gently advanced towards the mediastinum. Keeping the tip in constant view, the introducer is then used to gently dissect the pleura and mediastinal tissues off the undersurface of the sternum by using an anterior to posterior or "pawing" motion. When the tunnel extends for about 1 centimeter then the introducer may be moved in a side-to-side motion in order to enlarge the tunnel. Forward progress should always proceed by using the anterior to posterior motion since this decreases the risk of perforating the pericardium. The introducer should never be simply pushed forward and the tunnel should be wide enough to ensure good visibility. It cannot be stressed enough that the tip of the introducer should be kept in view at all

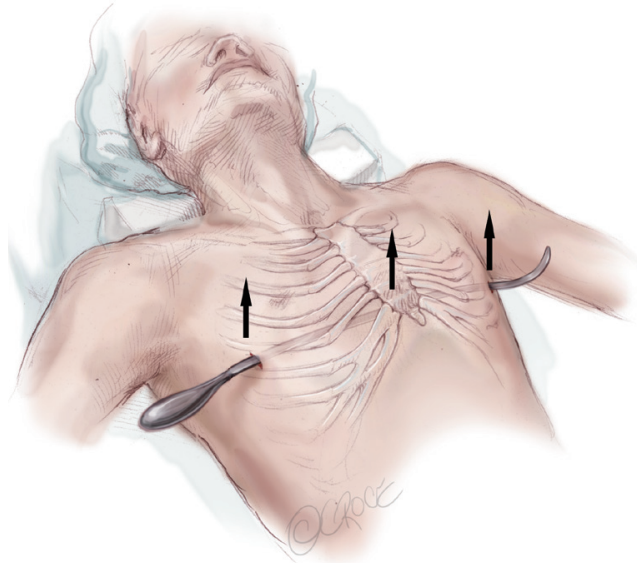

Figure 7 Shows sternal elevation using the introducer in order to correct the deformity, loosen up the anterior chest wall and stretch the ligaments. This greatly facilitates bar rotation, decreases pressure on the bar and minimizes bar displacement. This maneuver should be repeated several times.

times. When the left pleural cavity has been entered, the introducer is advanced up to the " $\mathrm{X}$ " on the left side of the chest and gently pushed through the intercostal space and then slowly advanced out of the subcutaneous tunnel. In order to prevent stripping the intercostal muscles during advancement, the assistant should insert a hook through the tip of the introducer when it first appears at the " $\mathrm{X}$ " on the left side. The assistant then pulls upward on the hook in an anterior direction while the surgeon pushes on his end in a transverse direction (Figure 6).

Once the introducer is in place it is lifted on both sides in an anterior direction to elevate the sternum out of its depressed position, while simultaneously pressing down on the lower chest wall and on any uneven protrusions. This molding process is repeated several times in an attempt to remodel the sternum by causing it to bow anteriorly. The lifting and molding maneuver decreases the corrective force which consequently decreases post-operative pain, decreases the risk of bar displacement, and need for re-shaping the bar after rotation (Figure 7).

Umbilical tape is attached to the hole in the tip of the introducer which is then slowly pulled back out of the chest under direct thoracoscopic visualization. The tape is cut off the introducer and attached to the previously selected and appropriately bent pectus bar. The bar is gently guided through the substernal tunnel with the convexity facing posteriorly by applying gentle traction on the umbilical tape. The bar insertion must also be performed under direct 

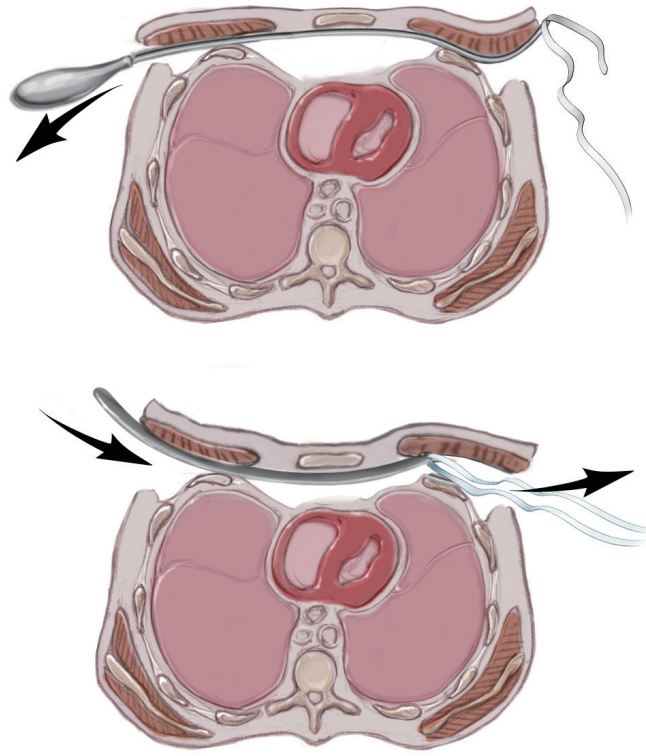

Figure 8 After the chest wall elevation procedure, umbilical tape is attached to the introducer which is then slowly withdrawn from the chest cavity under thoracoscopic guidance. When the introducer is out of the chest, the tape is cut off and attached to the pectus bar which is then slowly pulled through the media stinal tunnel with the convexity facing posteriorly under thoracoscopic control.

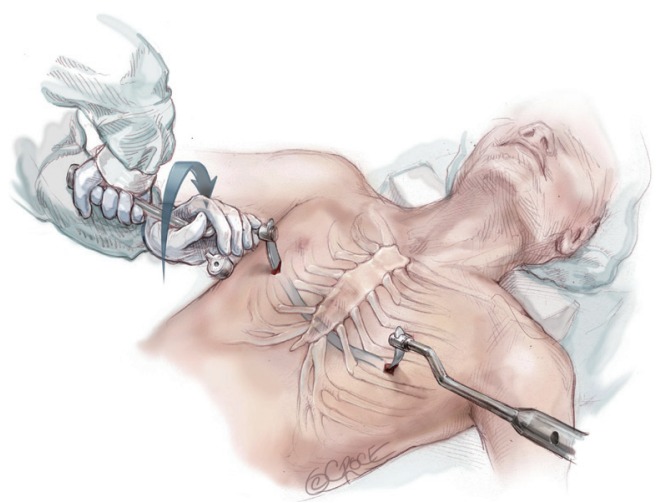

Figure 9 Shows how the pectus bar is rotated 180 degrees using the instrument specially designed for this purpose. The "bar flipper" should be applied close to the tip of the bar so that it can be easily removed once the bar has been rotated. A flipper may be applied to each end to facilitate rotation.

thoracoscopic visualization to avoid injuring the heart or pericardium (Figure 8).

Once the bar is in place with an equal amount of the bar protruding on each side, it is rotated 180 degrees using a bar flipping tool. The bar may be turned clockwise or counter- clockwise depending on whether there is more depression superiorly or inferiorly. After the bar is rotated into its correct position, it should be evaluated for proper fit against the chest wall and adjusted as needed with a bar bending tool to achieve a slightly loose fit. If the bar is too loose it will protrude and may be less stable, and if it is too tight it will cause persistent pain, rib erosion and calcification around the bar (Figure 9).

Fixation of the bar to the thorax is essential to prevent bar displacement. Typically a stabilizer is attached to the left side of the bar and secured to the bar with non-absorbable suture by engaging the grooves on the end of the bar to prevent slippage. Several absorbable sutures are also placed between the fascia of the lateral chest wall and the holes in the bar and stabilizer. If two bars are placed, the stabilizers are placed opposite each other. Stabilizers are important for long-term bar stability.

Through the right incision, several pericostal absorbable monofilament (polydioxanone) sutures are placed around the bar and the underlying rib where they intersect. Fixation to at least two separate ribs is crucial to improve stability of the bar and resist rotational forces postoperatively. All pericostal sutures should be placed under direct thoracoscopic visualization. The pericostal sutures are especially important for short-term bar stability (Figure 10).

Before closing the skin incisions, a final inspection of the mediastinum, heart, lungs, bar, and pericostal sutures is prudent to ensure hemostasis. The $\mathrm{CO}_{2}$ insufflation is discontinued and the anesthesiologist re-inflates the lungs. A three layered closure with absorbable suture is recommended to provide maximum coverage over the bar and stabilizer. While the incisions are being closed, the insufflation tubing attached to the thoracic trocar is divided and the proximal end is placed under water. Additionally, the patient is placed in the Durant position and placed on positive end-expiratory pressure $(5 \mathrm{mmHg})$ to maximize evacuation of air from the thoracic cavity. Prior to removing the trocar, the surgeon must ensure that there is no air remaining in the chest cavity (Figure 11).

\section{Post-operative management}

The anesthesiologist should be keenly aware of signs of pain during emergence from anesthesia. Every effort to achieve a smooth emergence from anesthesia is necessary to block the pain cascade and prevent the patient from excessive motion postoperatively. In the absence of bleeding concerns, a dose of ketorolac is given intravenously before the end of the 


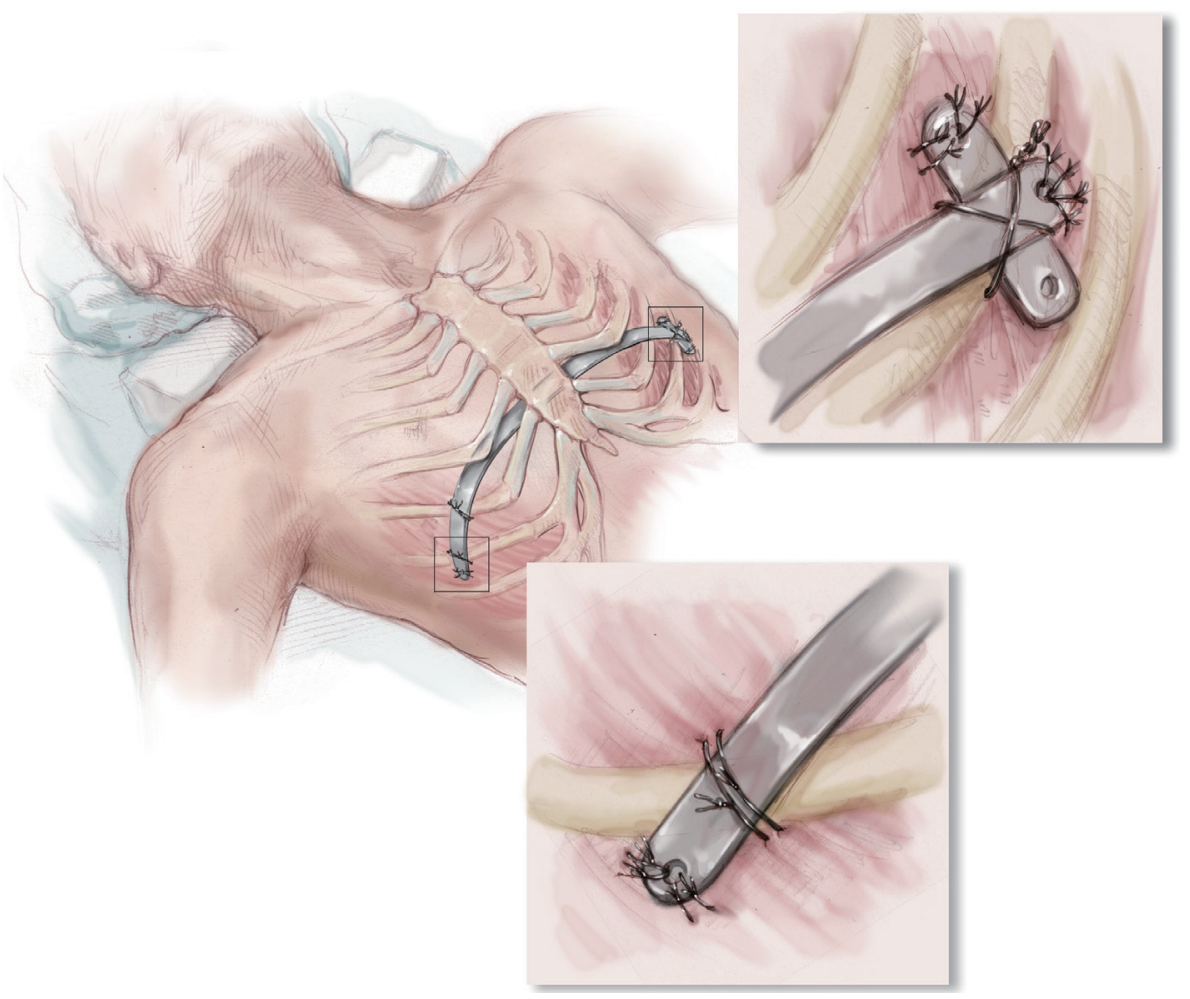

Figure 10 Bar fixation is essential to prevent displacement and is accomplished by applying a stabilizer on the left and multiple pericostal sutures on the right. The stabilizer is attached to the bar with wire or fiberwire suture. Sutures are also placed between the holes in the stabilizer and the pre-muscular fascia. Multiple pericostal sutures consisting of zero PDS are placed around the bar and ribs on the right side at the point where the bar crosses each rib.

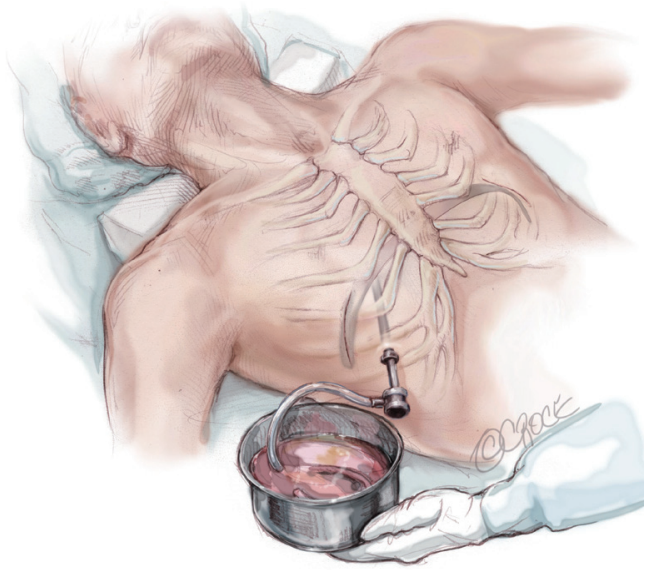

Figure 11 After the incisions are closed the pneumothorax is evacuated by cutting the insufflation tubing and placing the proximal end under water. The operating table is placed in Durant position and the anesthesiologist applies positive pressure ventilation until no more air bubbles escape from the tubing. The trocar is then pulled while the anesthesiologist applies positive pressure. procedure. Local anesthesia may be administered via liberal infiltration around the incisions, intercostal nerve blocks, or On-Q catheters. A patient controlled analgesia (PCA) pump using morphine or fentanyl is started in the operating room and transitioned to oral pain medication over the next 2-3 days. Other strategies to control postoperative pain include oral non-steroidal anti-inflammatory drugs (NSAIDs), muscle relaxants, and sedatives. Laxatives and stool softeners are given to prevent constipation and IV fluids and histamine-receptor 2 blockers are given to decrease the side-effects of the NSAID on the gastro-intestinal tract and kidneys. Recently one dose of methadone has been found to be effective when given at the end of surgery by the group from Phoenix (30).

Vigorous pulmonary toilet using an incentive spirometer (ICS) is started hours after surgery and continued for several weeks to prevent atelectasis and pneumonia. Respiratory therapists are helpful to provide directions for proper use of the ICS. Using the ICS also helps to expand the chest and loosen the ligaments which decreases the pressure and thus 


\begin{tabular}{lc}
\hline Table 1 Early postoperative complications & \\
\hline Complication & Number of Cases \\
\hline Pneumothorax & $3.8 \%(n=56)$ \\
\hline Drug reaction & $2.9 \%(n=43)$ \\
\hline Suture site infection & $1.9 \%(n=18)$ \\
\hline Pneumonia & $0.9 \%(n=13)$ \\
\hline Pleural effusion (req drainage) & $0.9 \%(n=13)$ \\
\hline Pericarditis & $0.6 \%(n=9)$ \\
\hline Hemothorax & $0.3 \%(n=5)$ \\
\hline Temporary paralysis & $0.1 \%(n=2)$ \\
\hline Cardiac perforation & $0.0 \%(n=0)$ \\
\hline Death & $0.0 \%(n=0)$ \\
\hline
\end{tabular}

Table 2 Late post-operative complications

\begin{tabular}{lc} 
Complication & Number of Cases \\
\hline A. Bar displacements requiring revision & $3.7 \%(n=54)$ \\
B. Over-correction & $3.1 \%(n=47)$ \\
C. Bar allergy & $2.7 \%(n=39)$ \\
D. Wound infection & $1.5 \%(n=22)$ \\
C. Recurrence & $0.9 \%(n=13)$ \\
\hline
\end{tabular}

the pain. Ambulation is started on the first postoperative day with the help of the physical therapists.

The patients are instructed to lie on their back and avoid any pressure on the sides of their chest for the first 6 weeks.

A portable chest $\mathrm{X}$-ray is obtained on the first postoperative day to check for pneumothorax, pleural effusion, and bar position. On the third postoperative day a posterior-anterior and lateral chest $\mathrm{X}$-ray is obtained in the X-ray department to again check for bar position, pneumothorax and pleural effusion.

When the patients are able to take all of their medications orally and ambulate unassisted they are discharged home, usually on postoperative day 4 or 5 .

At home, the patients are encouraged to walk as much as possible and do deep breathing exercises using the ICS multiple times a day. The oral narcotics, muscle relaxants, and sedatives are typically weaned off in the first one to two weeks. Oral anti-inflammatories may be required for another 1 to 2 weeks or more. Patients may return to school after 3 weeks but are not permitted to play sports or do heavy lifting for 6 weeks. At that time, if all is well, they may slowly resume normal activities and by 3 months they may participate in competitive sports.

\section{Results}

Early complications occurring before discharge from hospital in 1,463 primary repairs performed between 1987 and 2012 are listed in (Table 1).

These complications have decreased in frequency since the early learning period. Pneumothorax is usually due to incomplete evacuation of the $\mathrm{CO}_{2}$, since a leak in the lung parenchyma should only occur if adhesions to the lung need to be lysed. Drug reactions can be minimized by careful history taking. Suture site infections can be minimized by careful antiseptic technique and prophylactic antibiotics. Pneumonia can be prevented by careful preoperative examination to ensure that the patient is healthy and postoperatively by encouraging hourly use of ICS. Pericarditis should be a rare event due to routine pre-op metallic allergy testing and the use of titanium bars when nickel or other metallic allergy is suspected. Hemothorax is minimized by avoiding injury to the intercostal and internal mammary arteries and checking that there is no bleeding before pulling the trocar at the end of the procedure. Temporary paralysis was due to the epidural in two cases and this method of pain control was subsequently abandoned.

Late post-operative complications are listed in (Table 2).

Bar displacements presented an initial challenge and occurred in $15 \%$ of the patients in the first few years when the only method for bar stabilization was to create a soft tissue bed for the bar with mattress sutures. When stabilizers were developed, the rate of displacement dropped to $5 \%$, and with the addition of pericostal sutures, the displacement rate fell to $1 \%$.

Over-correction tends to occur in patients with a very deep and asymmetric deformity and a narrow chest. It is very important that these patients do deep breathing with breath holding exercises twice every day in order to open up the chest and make room for the cartilages. A carinatum brace may be applied if the over-correction does not improve after one year.

Bar allergy complications have been minimized by the use of routine pre-operative metal allergy testing and using titanium bars when an allergy to any of the components of stainless steel is proven or suspected. If the patient develops allergic complications after bar insertion, prednisone 


\begin{tabular}{lc} 
Table 3 Results & \\
\hline Total number post bar removal & $n=1,385$ \\
\hline Excellent result & $90.5 \%(n=1,254)$ \\
\hline Good result & $7.8 \%(n=108)$ \\
\hline Fair result & $0.5 \%(n=7)$ \\
\hline Poor result & $0.4 \%(n=5)$ \\
Failed result & $0.8 \%(n=11)$ \\
\hline
\end{tabular}

given until the ESR and CRP return to normal is usually successful, but if symptoms recur then removal of the stainless steel bar may be required.

Recurrence is inversely related to the length of time the bar remains in place (16). The shorter the time the bar is in place, the higher the recurrence rate. Since the duration of bar placement was increased to 3 years before removal there have been no recurrences. Patients are advised to do deep breathing exercises every morning and evening, in order to open up the entire chest and loosen up the chest wall ligaments. Patients are also encouraged to participate in exercises such as running and swimming starting 6 weeks after repair.

A review of our prospective database shows a good to excellent outcome in $98 \%$ of patients, where an excellent result implies a normal chest and a good result shows minimal residual depression. Most of the failures occurred during the learning curve in the first few years when the bar was too soft and it was removed too soon (Table 3).

The results of cardiac and pulmonary function studies show that there is an immediate beneficial effect from the release of right heart compression, giving rise to an increase in stroke volume and cardiac output, and a more gradual improvement of pulmonary function which is especially notable after bar removal $(22,31,32)$.

There has been a huge shift in the age at which patients are scheduled for pectus excavatum repair. From 1949 until 2000, the surgical literature recommended that children have the open pectus procedure before going to school (6,33-35). However, after an article by Haller and colleagues drew attention to the effects of too early and extensive open procedures (36), the median age shifted to 14 years. This shift was further accentuated by the introduction of MIRPE procedure since it could be done at any age (37). In addition, there have been reports in the literature of patients in their late 40's and 50's developing cardiopulmonary failure with no pathology other than pectus excavatum to account for their incapacitation (38).
As a result, there are now numerous reports of successful repair via the Nuss procedure in older adult patients $(24,29,38,39)$.

\section{Bar removal}

Bar removal should be scheduled between 2 to 4 years and done under general endotracheal anesthesia with PEEP. The EKG monitor should be audible. The bar should be mobilized at both ends, the stabilizer is removed, and the bar slowly straightened out with the bar flippers. When it is straight enough to slide out, it should first be slightly rotated to see the effect on the EKG. If the EKG remains stable then it should be slowly withdrawn from the chest. Local anesthetic is injected and the incisions closed in layers and pressure dressings applied. A chest X-ray is taken in the recovery room to check for pneumothorax.

\section{Future}

Since the introduction of the Minimally Invasive Pectus Repair, there has been a dramatic increase in the number of patients presenting for repair. There has also been a dramatic increase in the number of papers published in the medical literature-300 papers in the decade before the Nuss bar procedure and over 600 in the decade after. The increase in the number of pectus excavatum patients presenting for repair has allowed for a large increase in the number of studies of cardio-pulmonary function, showing that these patients do suffer from right heart compression and pulmonary restriction, which is significantly improved after repair $(22,24,29,31,32,37-39)$. It should be noted that a $10 \%$ reduction in pulmonary function studies is significant as it represents a full standard deviation below normal. Although these patients may be asymptomatic at rest, they are not able to compete with their peers. In addition, there are now more reports of patients in their $4^{\text {th }}$ and $5^{\text {th }}$ decade becoming incapacitated due to their cardiopulmonary compression (39).

In the decade since the MIRPE procedure was first published, many modifications have been made to make the procedure safer and more efficient including: addition of thoracoscopy, stabilizers, pericostal sutures, sternal elevation before tunneling, instruments designed specifically for the procedure, prophylactic antibiotics, and optimization of pain control (37). It is anticipated that this trend will continue and that the procedure and instruments will constantly be improved $(17,18)$.

Even the open procedure has benefitted as it has been 
modified from the original radical resection Ravitch procedure to a much less aggressive minimal cartilage resection open procedure $(40,41)$.

Centers of excellence will develop in which there will be a team of well-trained surgeons, anesthesiologists, nurses, pain specialists, physical therapists and respiratory therapists who will provide expert care with even fewer complications and outstanding results.

The instruments will be constantly improved both as a result of new ideas but also because of the discovery of new products which are more suitable for the procedure such as new alloys and bio-absorbable materials.

New approaches to the problem will also appear, as exemplified by Klobe's Vacuum Bell, which is now finding a place as the treatment of choice in younger patients with a soft and malleable chest who have a mild to moderate deformity $(19,20)$. At one time there was only one procedure available for patients with pectus excavatum and that was the Ravitch procedure. Now, there is a spectrum of techniques extending from the non-operative Vacuum Bell treatment for mild and moderate cases, to the MIRPE procedure for more severe cases, to the modified open procedure for the very severe and recurrent cases, as well as combined open and closed procedures $(42,43)$.

Other new ideas are presently at the experimental stage, including Bardaji's Pectus Up procedure, in which a screw is used to pull the sternum up, and Harrison's Magnetic MiniMover Procedure, in which magnets are used to pull the sternum up over a 2 year time frame (44).

In the future, the treatment of pectus excavatum will be more proactive as primary care doctors and parents begin to fully appreciate the range of treatment methods available for treating chest wall malformations. The patients will be referred at an earlier age, which will allow the conservative treatment options to be more successful. This may cause a slight decline in the number of patients requiring surgical repair, but in those patients requiring surgery, the timing will be optimized.

\section{Acknowledgements}

Thanks to Trisha Arnel, Executive Secretary.

\section{Footnote}

Conflicts of Interest: Dr. Nuss has consultation and royalty with Zimmer Biomet. Dr. Kelly and Dr. Obermeyer have consultation agreements with Zimmer Biomet.

\section{References}

1. Bigelow HJ. Insensibility during Surgical Operations Produced by Inhalation. Boston Med Surg J 1846;35:309-17.

2. Meade RH. A history of thoracic surgery. Springfield, Ill. : C.C. Thomas, 1961.

3. Meyer L. Zur chirurgischen Behandlung der angeborenen Trichterbrust. Berl Kiln Wschr 1911;48:1563-6.

4. Sauerbruch F. Die Chirurgie der Brustorgane, Vol. 1, Julius. Berlin: Springer. 1920:437.

5. Sauerbruch F. Operative Beseitigung der angeborenen Trichterbrust. Deutsche Zeitschrift Für Chirurgie 1931;234:760-4.

6. Ravitch MM. The Operative Treatment of Pectus Excavatum. Ann Surg 1949;129:429-44.

7. Gellis SS. Pediatric Notes. The Weekly Pediatric Commentary 1996;20:99-100.

8. Paltia V, Parkkulainen KV, Sulamaa M, et al. Operative technique in funnel chest; experience in 81 cases. Acta Chirurgica Scandinavica 1959;116:90-8.

9. Adkins PC, Blades B. A stainless steel strut for correction of pectus escavatum. Surg Gynecol Obstet 1961;113:111-3.

10. Kelley SW. Surgical Diseases of Childhood. 3rd Ed. The CV Mosby Co. 1929;1:537.

11. Haller JA Jr. Pediatric Surgery. Vol 1, p 147, 4th Edit. Y-B Med Publ. 1986 ED Welch KJ, Randolph JG, Ravitch MM, et al.

12. Wesson DE. Pediatric Surgery. Vol 1, p 245-247, 5th Edit Y-B Med Publ. 1998. ED by O’Neil, Rowe, Grosfeld, et al.

13. Am Heart Assoc \& Am Acad Peds. Textbook of Pediatric Advanced Life Support. Fig 2.7.

14. Nuss D, Kelly RE Jr, Croitoru DP, et al. A 10-year review of a minimally invasive technique for the correction of pectus excavatum. J Pediatr Surg 1998;33:545-52.

15. Croitoru DP, Kelly RE Jr, Goretsky MJ, et al. Experience and modification update for the minimally invasive Nuss technique for pectus excavatum repair in 303 patients. $\mathrm{J}$ Pediatr Surg 2002;37:437-45.

16. Nuss D. Minimally invasive surgical repair of pectus excavatum. Semin Pediatr Surg 2008;17:209-17.

17. Park HJ, Kim KS, Lee S, et al. A next-generation pectus excavatum repair technique: new devices make a difference. Ann Thorac Surg 2015;99:455-61.

18. Nuss D, Kelly RE. The Minimally Invasive Repair of Pectus Excavatum. Operative Techniques in Thoracic and Cardiovascular Surgery: A Comparative Atlas 2014;19:324-47.

19. Cohee AS, Lin JR, Frantz FW, et al. Staged management 
of pectus carinatum. J Pediatr Surg 2013;48:315-20.

20. Haecker FM. The vacuum bell for conservative treatment of pectus excavatum: the Basle experience. Pediatr Surg Int 2011;27:623-7.

21. Koumbourlis AC, Stolar CJ. Lung growth and function in children and adolescents with idiopathic pectus excavatum. Pediatr Pulmonol 2004;38:339-43.

22. Sigalet DL, Montgomery M, Harder J, et al. Long term cardiopulmonary effects of closed repair of pectus excavatum. Pediatr Surg Int 2007;23:493-7.

23. Maagaard M, Tang M, Ringgaard S, et al. Normalized cardiopulmonary exercise function in patients with pectus excavatum three years after operation. Ann Thorac Surg 2013;96:272-8.

24. Chao CJ, Jaroszewski DE, Kumar PN, et al. Surgical repair of pectus excavatum relieves right heart chamber compression and improves cardiac output in adult patients-an intraoperative transesophageal echocardiographic study. Am J Surg 2015;210:1118-24; discussion 1124-5.

25. St Peter SD, Juang D, Garey CL, et al. A novel measure for pectus excavatum: the correction index. J Pediatr Surg 2011;46:2270-3.

26. Pilegaard HK, Licht PB. Can absorbable stabilizers be used routinely in the Nuss procedure? Eur J Cardiothorac Surg 2009;35:561-4.

27. Rygl M, Vyhnanek M, Kucera A, et al. Technical innovation in minimally invasive repair of pectus excavatum. Pediatr Surg Int 2014;30:113-7.

28. Yoshida A, Uemura S, Yamamoto M, et al. Correlation of asymmetric chest wall deformity and growth in patients with pectus excavatum. J Pediatr Surg 2013;48:771-5.

29. Jaroszewski DE, Johnson K, McMahon L, et al. Sternal elevation before passing bars: a technique for improving visualization and facilitating minimally invasive pectus excavatum repair in adult patients. J Thorac Cardiovasc Surg 2014;147:1093-5.

30. Singhal NR, Jones J, Semenova J, et al. Multimodal anesthesia with the addition of methadone is superior to epidural analgesia: A retrospective comparison of intraoperative anesthetic techniques and pain management for 124 pediatric patients undergoing the Nuss procedure. J Pediatr Surg 2016;51:612-6.

31. Lawson ML, Mellins RB, Tabangin M, et al. Impact of pectus excavatum on pulmonary function before and after repair with the Nuss procedure. J Pediatr Surg 2005;40:174-80

32. Kelly RE Jr, Mellins RB, Shamberger RC, et al. Multicenter study of pectus excavatum, final report: complications, static/exercise pulmonary function, and anatomic outcomes. J Am Coll Surg 2013;217:1080-9.

33. WELCH KJ. Satisfactory surgical correction of pectus excavatum deformity in childhood; a limited opportunity. J Thorac Surg 1958;36:697-713.

34. Ravitch MM in Pediatric Surgery 4th Edition, 1986,Chapter 57, p574. ED by Welch KJ, Randolph JG, Ravitch MM, O’Neil JA, Rowe MI. Publ by YBMP.

35. Shields TW. Chest Wall Deformities in General Thoracic. Surgery, 3rd Edition. 1989:515-23.

36. Haller JA Jr, Colombani PM, Humphries CT, et al. Chest wall constriction after too extensive and too early operations for pectus excavatum. Ann Thorac Surg 1996;61:1618-24; discussion 1625.

37. Kelly RE, Goretsky MJ, Obermeyer R, et al. Twentyone years of experience with minimally invasive repair of pectus excavatum by the Nuss procedure in 1215 patients. Ann Surg 2010;252:1072-81.

38. Pilegaard HK. Extending the use of Nuss procedure in patients older than 30 years. Eur J Cardiothorac Surg 2011;40:334-7.

39. Kragten HA, Siebenga J, Höppener PF, et al. Symptomatic pectus excavatum in seniors (SPES): a cardiovascular problem? : A prospective cardiological study of 42 senior patients with a symptomatic pectus excavatum. Neth Heart J 2011;19:73-8.

40. Lopushinsky SR, Fecteau AH. Pectus deformities: a review of open surgery in the modern era. Semin Pediatr Surg 2008;17:201-8.

41. Fonkalsrud EW. Open repair of pectus excavatum with minimal cartilage resection. Ann Surg 2004;240:231-5.

42. Al-Assiri A, Kravarusic D, Wong V, et al. Operative innovation to the "Nuss" procedure for pectus excavatum: operative and functional effects. J Pediatr Surg 2009;44:888-92.

43. Varela P, Torre M. Thoracoscopic cartilage resection with partial perichondrium preservation in unilateral pectus carinatum: preliminary results. J Pediatr Surg 2011;46:263-6.

44. Harrison MR, Gonzales KD, Bratton BJ, et al. Magnetic mini-mover procedure for pectus excavatum III: safety and efficacy in a Food and Drug Administration-sponsored clinical trial. J Pediatr Surg 2012;47:154-9.

Cite this article as: Nuss D, Obermeyer RJ, Kelly RE. Nuss bar procedure: past, present and future. Ann Cardiothorac Surg 2016;5(5):422-433. doi: 10.21037/acs.2016.08.05 